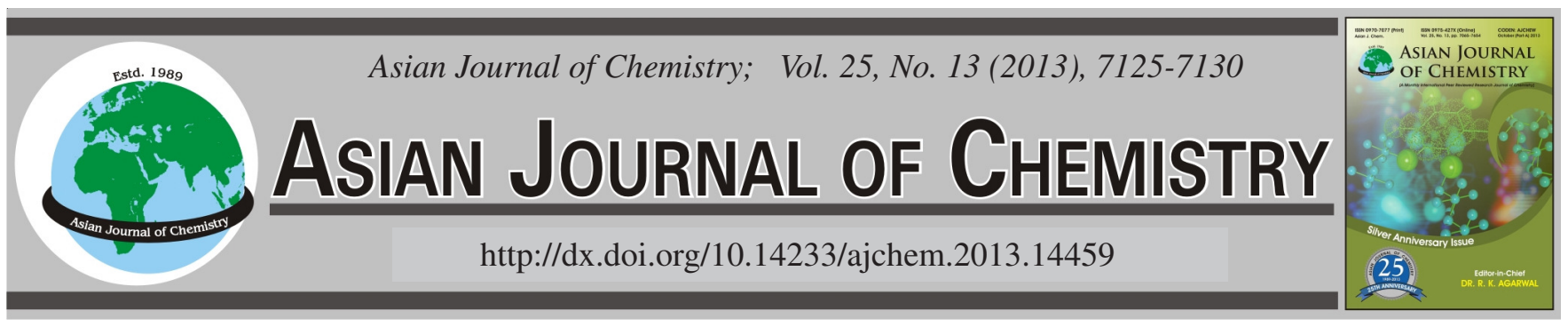

\title{
Microwave-Assisted Extraction and Nitric oxide and Superoxide Attenuation Potential of Polyphenolics from Bauhinia variegata
}

\author{
Shumaila Anwar, Nazish Jahan ${ }^{*}$, Khalil-Ur-Rahman, Shaukat Ali and Saba Aslam
}

Department of Chemistry and Biochemistry, University of Agriculture, Faisalabad, Pakistan

*Corresponding author: E-mail: nazishjahanuaf@yahoo.com

\begin{abstract}
An emerging microwave-assisted extraction has many advantages over conventionally used extraction techniques for the extraction of antioxidants. This research had been designed to investigate the efficiency of microwave assisted technique for the extraction of antioxidant polyphenols from different parts of Bauhinia variegata. Polyphenols were extracted by conventional (soxhlet, reflux) and emerging techniques (microwave-assisted extraction method) by employing solvents of different polarity. Total phenolic contents were measured by Folin-Ciocalteu method. Highest total phenolic contents were observed in the stem extract of $B$. variegata using Soxhlet technique but microwave-assisted extraction was found to be the fastest method for the extraction of phenolic contents. Nitric oxide and superoxide attenuation potential were evaluated by nitric oxide and super oxide radical attenuation assays. DPPH attenuation, reducing power, antioxidant activity in linoleic acid was also evaluated. Highest antioxidant potential was found in stem bark extract of $B$. variegata followed by flower and leave.
\end{abstract}

Key Words: Microwave-assisted extraction, Antioxidant potential, Nitric oxide attenuation potential, Super oxide attenuation potential.

ᄂ - - - - - - - - - - - - - - - - - - - - - - - - - - -

\section{INTRODUCTION}

Free radicals are very important for human body. Reactive nitrogen and reactive oxygen species that produce are integral part of many biological processes. They are involved in the defensive mechanism of the body. Excess production of these free radicals can be lethal ${ }^{1}$. Surplus amount of nitric oxide is linked with numerous diseases. Superoxides anion $\left(\mathrm{O}_{2}^{-}\right)$is generated through many processes and damage biomolecules that may result in lipid peroxidation, cardiac diseases and many other disorders. In living systems, production of free radicals is balanced with the help of antioxidative defensive mechanism of the body ${ }^{2}$.

Plant materials (leaves, flowers or fruits) show their antioxidant potential and radical scavenging activity as they possess a combination of phenolic compounds (flavonoids, anthocyanosides, tannins, catechins) which act as antioxidants. These phenolic compounds are derivatives of hydroxycinnamate, hydroxycoumarins, flavanones, flavonols, flavones, proanthocyanidins (tannins), anthocyanins, aurones, hydroxyl stilbenes and many others. Their antioxidant activity is due to their powerful chain-breaking activities and also their free radical scavenging capacity, thus preventing cells toward the toxic effects of free radicals. The main focus of investigators is now on the antioxidant potential of medicinal plants because they have maximum antioxidant activities and many health benefits ${ }^{3}$.

The extraction of polyphenolic antioxidant from plant material is based on the best selection of solvents, temperature conditions and agitation to maximize the solubility of the antioxidant polyphenols and enhance the mass transfer ${ }^{3}$. The conventional technique needs longer extraction time so there is great risk of thermal degradation for phyto-constituents. There was a need for safe, cost effective source of antioxidants to neutralize the free radical burden in the body. A promising microwave-assisted extraction has many advantages over conventionally used techniques. It saves time, energy and organic solvents. Comparatively innovative microwave assisted extraction has getting attention of scientists.

The aim of the current study was to examine the effect of various extraction parameters on the extractability of polyphenols and the antioxidant potential in different parts of Bauhinia variegata. B. variegata belongs to family Caesalpiniaceae and Genus Bauhinia. This plant has valuable medicinal importance. Its stem bark is utilized as tonic, anthelmintic, astringent, antioxidant and antidiarrheal ${ }^{4}$. Due to great medicinal importance, its different parts (leaves, stem, flowers) are used for the extraction of polyphenols with conventional (reflux, Soxhlet) and improved (microwave-assisted extraction) techniques. Solvents of different polarity (methanol, ethyl acetate, water) were used 
for extraction. Then the extracts were compared for their total phenolic contents and antioxidant activity.

\section{EXPERIMENTAL}

Numerous chemicals and reagents were used to accomplish the scope of current research. These were ethylene diamine tetra acetate (EDTA), 2,2-diphenyl-1-picrylhydrazyl (DPPH), butylated hydroxytoluene (BHT), nitrobluetetrazolium reagent (NBT), coupling reagent [ $N$-(1-naphthyl)ethyl-enediaminedihydrochloride], ferrous chloride, ammonium thiocyanate, trichloroacetic acid, gallic acid, sodium tungstate, sodium molybdate, lithium sulphate, potassium ferricyanide, hydrochloric acid, bromine water, ascorbic acid, hydroxylamine hydrochloride, sodium carbonate, disodium hydrogen phosphate, sodium dihydrogen phosphate, sodium nitroprusside, sulphonamide, phosphoric acid, ferric chloride, linoleic acid, Tween 80, nitric acid, methanol, ethyl acetate, Folin-Ciocalteu reagent and Griess reagent.

Collection of plant material: Fresh leaves stem and flowers of Bauhinia variegata were collected from Botanical Garden of University of Agriculture, Faisalabad. Fresh parts of Bauhinia variegata were shade dried and grinded to get powdered form.

Preparation of plant extracts: Extracts were prepared by using different techniques (soxhlet, reflux, microwave) and solvents (methanol, ethyl acetate, water) of different polarities. On the whole, 24 extracts were prepared and they were compared for their polyphenolic contents and antioxidant activity.

Soxhlet technique: Dry powder plant material (30 g) of each part was placed in thimble and extracted with methanol and ethyl acetate $(300 \mathrm{~mL})$ separately for $6 \mathrm{~h}$ in a Soxhlet apparatus. Extracts were concentrated and stored in a refrigerator for further analysis.

Reflux technique: Dry powder plant material (30 g) of each part was extracted with methanol, ethyl acetate and water $(100 \mathrm{~mL})$ separately for $1 \mathrm{~h}$ in a reflux apparatus. Extracts were filtered and concentrated using water bath. Concentrates were stored in a refrigerator for further analysis.

Microwave-assisted extraction technique: Microwave assisted extraction was performed with an oven specific for this function. Two different solvents were used for extraction (water, methanol). By varying experimental conditions (material to solvent ratio, temperature, irradiance time) parameters were optimized to get maximum output. Optimum conditions for water as solvent: irradiance time 2 and $4 \mathrm{~min}$ and material to solvent ratio 1:20. Same conditions were also found optimum for methanol but 4 min irradiation time was not found suitable. Extracts obtained were filtered, concentrated and stored for further consideration.

Determination of total polyphenols: Total polyphenolic contents were determined in extracts of Bauhinia by FolinCiocalteu assay ${ }^{5}$. All extracts prepared above were assessed for polyphenolic contents. Quantification was done with respect to the standard (gallic acid). Different concentrations of gallic acid were prepared $(0.01-0.10 \mathrm{mg} / \mathrm{mL})$. Gallic acid $(1 \mathrm{~mL})$ was mixed with Folin-Ciocalteu reagent $(5 \mathrm{~mL}, 10$ times diluted with distilled water) and sodium carbonate (4 $\mathrm{mL}, 20 \%)$. Same procedure was repeated with plant extracts
(1 mL). A blank solution was also prepared in similar way (without extract). The absorbance of consequential complex was assessed at $765 \mathrm{~nm}$ with UV-visible spectrophotometer. Calibration curve was plotted by taking the absorbance as a function of concentration. All experiments were repeated thrice. Total polyphenolic contents in plant extracts were calculated by the following formula.

Total phenolic contents $(\mathrm{mg} \mathrm{GAE} / \mathrm{g})=$

Unknown concn. of plant extract $\times$ volume of extract $(\mathrm{mL})$

\section{Weight of plant extract $(\mathrm{g})$}

The unknown concentration of plant extract calculated from standard curve in $\mathrm{mg} / \mathrm{mL}$.

Nitric oxide attenuation potential: Nitric oxide attenuation potential of plant extract was explored by the scheme of Govindarajan et al. ${ }^{6}$. Sodium nitroprusside solution $(5 \mathrm{mM})$ was prepared in phosphate buffer $(0.2 \mathrm{M}, \mathrm{pH} 7)$. Sodium nitroprusside $(1 \mathrm{~mL})$ in saline buffer was mixed with plant extracts $(2 \mathrm{~mL})$ of different concentrations $(0.02-0.1 \mathrm{mg} / \mathrm{mL})$ and incubated at room temperature for $0.5 \mathrm{~h}$. After $0.5 \mathrm{~h}$, incubated solution $(2 \mathrm{~mL})$ removed and added Griess reagent $(2 \mathrm{~mL})$. A parallel control was also treated alike (without extracts). The absorbance of resulting solutions were noted at $500 \mathrm{~nm}$. Quantization was done with standard BHT and ascorbic acid. All experiments were repeated thrice. Results were stated as percentage inhibition which was calculated by subsequent formula.

Inhibition $(\%)$ of nitric oxide $=\left[1-\frac{\text { Absorbance of sample }}{\text { Absorbance of control }}\right] \times 100$

Superoxide radical attenuation potential: Super oxide radical attenuation potential of plant extracts was find out as stated by Vaidya et al. ${ }^{7}$. Various concentrations of plant extracts were prepared (0.2-1.0 mg/mL). Experiment was carried out by taking plant extract $(2 \mathrm{~mL})$, sodium carbonate $(2 \mathrm{~mL}, 5$ $\%)$, nitro blue tetrazolium reagent $(0.8 \mathrm{~mL}, 150 \mu \mathrm{M})$ and EDTA $(0.6 \mathrm{~mL}, 0.5 \%)$. Same procedure was repeated with standards BHT and ascorbic acid $(2 \mathrm{~mL})$. Absorbance of resulting solutions was taken at $0 \mathrm{~min}$. The reaction was initiated when hydroxyl-amine hydrochloride $(0.8 \mathrm{~mL}, 1 \%)$ was added to the above solution. Reaction mixture was incubated for $5 \mathrm{~min}$ at $25^{\circ} \mathrm{C}$. The NBT reduction was noted at $560 \mathrm{~nm}$. A parallel control was also treated in the same way (without extract). All experiments were repeated thrice. Results were stated as percentage inhibition which was calculated by subsequent formula.

Inhibition $(\%)$ of super oxide $=\left[1-\frac{\text { Absorbance of sample }}{\text { Absorbance of control }}\right] \times 100$

DPPH attenuation potential: DPPH radical attenuation potential was assessed by the method described by Yen and Chen ${ }^{8}$. Various concentrations of plant extracts $(0.02-0.1 \mathrm{mg} /$ $\mathrm{mL}$ ) were prepared. Freshly prepared solution of $0.1 \mathrm{mM}$ DPPH $(1 \mathrm{~mL})$ was taken and added $(3 \mathrm{~mL})$ of these concentrations. These solutions were kept for $0.5 \mathrm{~h}$ in dark. Then absorbance was noted at $517 \mathrm{~nm}$ using UV-visible. Spectrophotometer. Quantization was done with standards (BHT, ascorbic acid). The solution without plant extract was used as control and run in similar way. All experiments were repeated 
thrice. Results were stated as percentage inhibition, which was calculated by subsequent formula.

Inhibition $(\%)$ of DPPH radical $=\left[1-\frac{\text { Absorbance of sample }}{\text { Absorbance of control }}\right] \times 100$

Measurement of reducing power: Reducing power was measured as stated by Yen and Chen ${ }^{8}$. Different concentrations $(0.2-1 \mathrm{mg} / \mathrm{mL})$ of plant extracts and standards (BHT and ascorbic acid) were prepared. Each concentration of plant extract and standards $(2 \mathrm{~mL})$ was mixed with phosphate buffer ( $2.5 \mathrm{~mL}, 0.2 \mathrm{M}$ and $\mathrm{pH}=6.6)$ and potassium ferricyanide $(2.5$ $\mathrm{mL}, 1 \%)$. The control was also prepared, containing same reaction reagents with the exception of plant extract. The reaction mixtures were incubated at $50{ }^{\circ} \mathrm{C}$ for $20 \mathrm{~min}$. The reaction was stopped by the addition trichloroacetic acid $(2.5$ $\mathrm{mL}, 1 \% \mathrm{w} / \mathrm{v})$. Then the mixture was centrifuged at $3000 \mathrm{rpm}$ for $10 \mathrm{~min}$. Supernatant layer $(2.5 \mathrm{~mL})$ of each reaction mixture was blended with water $(2.5 \mathrm{~mL})$ and ferric chloride $(0.5 \mathrm{~mL}$, $0.1 \% \mathrm{w} / \mathrm{v})$. Then the absorbance was recorded at $700 \mathrm{~nm}$. Increased absorbance of the mixture with increase in concentration reflects more reducing power of the sample.

Antioxidant potential in linoleic acid system: Antioxidant potential of various extracts against lipid peroxidation was assessed in linoleic acid system through ammonium thiocyanate assay ${ }^{9}$. Extract $(1 \mathrm{~mL})$ was added to linoleic acid emulsion $(5 \mathrm{~mL})$ and phosphate buffer (4 mL, $0.04 \mathrm{M}, \mathrm{pH} 7)$. Linoleic acid emulsion was made by mixing linoleic acid $(0.2804 \mathrm{~g})$, Tween 80 as emulsifier $(0.2804 \mathrm{~g})$ and phosphate buffer $(50 \mathrm{~mL})$ then the mixture was homogenized. The mixed samples were incubated at $37^{\circ} \mathrm{C}$ for $72 \mathrm{~h}$ to accelerate the oxidation process. After each $24 \mathrm{~h}, 2 \mathrm{~mL}$ of the incubated sample was taken out of it and $0.5 \mathrm{~mL}$ of $\mathrm{FeCl}_{2}(0.02 \mathrm{M})$ and $0.5 \mathrm{~mL}$ of $30 \%(\mathrm{w} / \mathrm{v})$ ammonium thiocyanate were added. The absorbance was noted at $500 \mathrm{~nm}$ to determine the amount of peroxides. Samples were analyzed after each $24 \mathrm{~h}$, waiting the day when control solution absorbance (without extract) reached utmost value. BHT and ascorbic acid were used as standards. All experiments were repeated thrice. Results were stated as percentage inhibition which was calculated by subsequent formula.

Inhibition $(\%)$ of lipid peroxidation $=\left[1-\frac{\text { Absorbance of sample }}{\text { Absorbance of control }}\right] \times 100$

Statistical examination: The results were stated as Mean \pm S.D.

\section{RESULTS AND DISCUSSION}

Results from current study lead to a better description of the antioxidant properties of different parts of Bauhinia variegata and revealed which of them are the best sources of dietary antioxidants.

Total phenolic contents: Polyphenols are natural antioxidant they possess the capacity to bind with free radicals and stop free radicals chain reactions. Thus they prevent us from many fatal disease ${ }^{10,11}$. Folin-Ciocalteu method was employed to examine the total phenolic contents. Many research workers described the use of Folin-Ciocalteu method to evaluate total phenolic contents ${ }^{11-13}$.
Various techniques and solvents can be utilized for the extraction of polyphenols from plants ${ }^{13}$. Choice of a technique and solvent may depend on the type of phenolic compound be supposed to be isolated. Type of extraction system significantly determined the extraction of polyphenols. Extraction efficiency of phenolics is affected by prolonged heating and hydrolysis ${ }^{14}$.

Results of total phenolic contents are illustrated in Table-1. They were evaluated from standard calibration curve of gallic acid (Fig. 1). Considerable amounts of total phenolic contents were determined in all parts of plant extracts. As seen in Table1, total phenolic contents of all parts of plant ranged from 400 to $950 \mathrm{mg} \mathrm{GAE} / \mathrm{g}$ of extract. Maximum total phenolic contents were found in stem of Bauhinia variegata followed by flower and leaves irrespective of the technique used. Soxhlet yielded highest phenolic contents $(950 \pm 0.01 \mathrm{mg} \mathrm{GAE} / \mathrm{g}$ ) followed by reflux $(850 \pm 0.05 \mathrm{mg} \mathrm{GAE} / \mathrm{g})$ and microwave $(720 \pm 0.06$ $\mathrm{mg} \mathrm{GAE} / \mathrm{g}$ ). Although soxhlet and reflux techniques yielded more polyphenols than microwave-assisted extraction but they are not suitable for extraction purposes as they are time and solvent consuming.

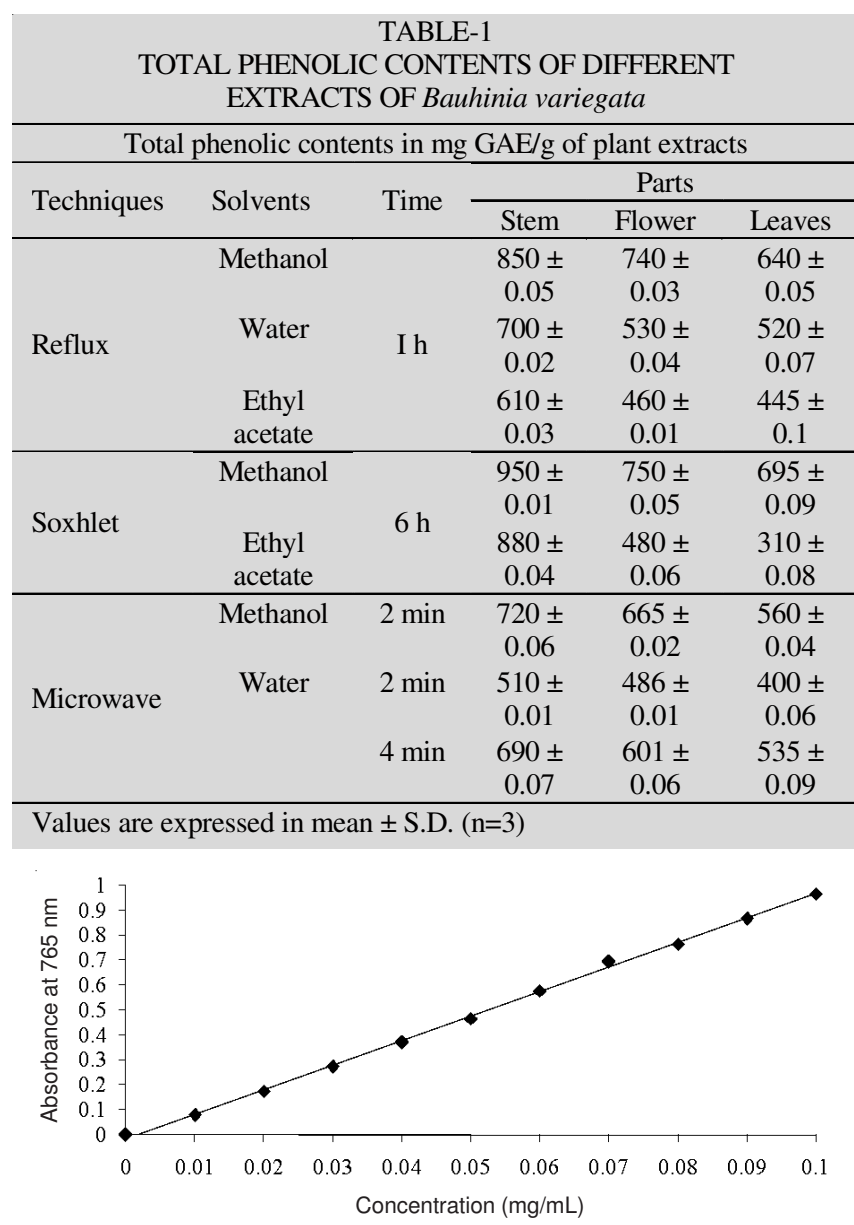

Fig. 1. Standard calibration curve between different concentrations of Gallic acid and absorbance at $765 \mathrm{~nm}$

Microwave-assisted extraction technique yielded comparable polyphenols in less time. Some other reported microwave-assisted extraction technique as most effective for the extraction of polyphenols from plants. It is better than Soxhlet and heat reflux extraction due to high extraction efficiency, short time for extraction and less energy consumption ${ }^{15}$. 
Extract yield and recovered antioxidant properties of plant material are also significantly depend on the nature of the solvent used. Polar solvents are mostly used for the extraction of polyphenols from plant materials ${ }^{13}$. In current research, methanol was found to be most useful solvent for the extraction of polyphenols followed by water and ethyl acetate irrespective of the technique used. Methanol yielded maximum polyphenols due to its high polarity and organic nature.

Determination of antioxidant potential: Various chemical in vitro assays have been developed to measure antioxidant capacities of plant products. A number of these assays depend on the generation of free radicals. Despite the recent popularity in antioxidant research, the lack of standardized assays to compare research results from different research groups has been a major challenge. Due to the complex nature of biological systems, there is no single universal method for measuring antioxidant capacity ${ }^{16}$.

Nitric oxide attenuation potential: Nitric oxide is a diffusible free radical that takes part in various biological processes such as vasodilatation, neuronal messenger, antitumor and antimicrobial activities. Surplus amount of free radicals (nitric oxide and superoxide) are linked with deleterious diseases. Our defensive biological system may not be adequate to counteract nitric oxide, superoxide and other reactive species. Sodium nitroprusside produces nitric oxides at physiological $\mathrm{pH}$, interact with oxygen to produce nitrite ions, which can be estimated using Griess reagent. The compounds that possess nitric oxide scavenging activity inhibit nitrite formation by competing with oxygen. This leads to decrease nitrite concentration in vitro ${ }^{17}$.

In the current investigation, antioxidant potential of Bauhinia variegata was evaluated by nitric oxide scavenging activity. Many previous studies recommended this assay for determination of antioxidant activity ${ }^{2}$. Results of percentage inhibition of nitric oxide radical for various extracts of Bauhinia variegata are shown in Fig. 2. The extracts were found to scavenge the nitric oxide radical at the various concentrations tested. According to these result, free radical scavenging activity of methanol extracts of all samples ranged from $25.89 \pm 0.062 \%$ to $72.26 \pm 1.23 \%$. Standards including ascorbic acid $(67.6 \pm 0.98 \%)$ and BHT $(72.26 \pm 1.23 \%)$ showed the highest percentage inhibition of nitric oxide radical as compared to that of plant extracts. Among parts of Bauhinia variegata, stem extract showed the highest attenuation power $(65.21 \pm 1.18 \%)$ followed by flower $(59.07 \pm 1.87 \%)$ and leave extract $(50.4 \pm 0.9 \%)$. Results were obtained in the following order; BHT $>$ ascorbic acid $>$ stem $>$ flower $>$ leaves.

Results of this study revealed that percentage inhibition of all the extracts increases with increase in concentration. These results were in agreement with Rajani and Ashok ${ }^{18}$. In current study, there was also found a significant correlation between concentration and percentage inhibition of nitric oxide radical. The extracts having greater total phenolic contents also showed greater nitric oxide inhibition ability. Thus from this research it is also concluded that total phenolics contents are related to nitric oxide scavenging ability.

Superoxide anion attenuation potential assay: Superoxide anions radicals are one of the most abundantly produced

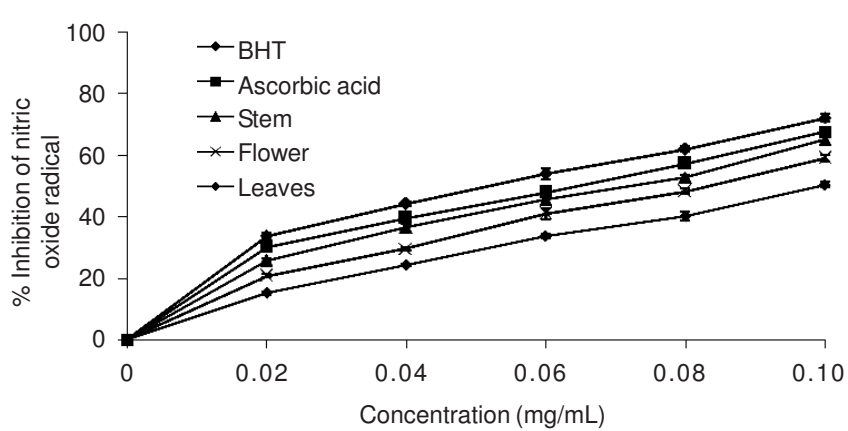

Fig. 2. Percentage inhibition of nitric oxide radical by different extracts of Bauhinia variegata

free radicals and are produced endogenously by flavoenzymes. Superoxide and hydrogen peroxide serve as precursors of singlet oxygen and hydroxyl ${ }^{19}$ radicals and indirectly prompt lipid oxidation. Superoxide radicals can be assessed by simple assay. In this they are produced by oxidation of hydroxylamine hydrochloride and judged by the reduction of nitro blue tetrazolium reagent. Nitro blue tetrazolium reagent converted in to formazan by reduction when there is no antioxidant. Generation of formazan can be assessed with spectrophotometer at 560 $\mathrm{nm}$. Reduction in absorbance in the presence of extract reflects antioxidant potential ${ }^{20}$.

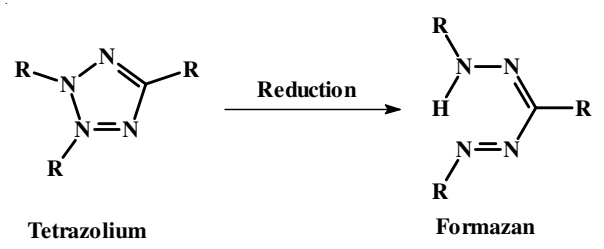

In the present study antioxidant potential of Bauhinia variegata was determined by using super oxide radicals scavenging assay. It is one of most popular methods for evaluation of antioxidant activity. Many previous studies were reported about the use of this assay for the investigation of antioxidant activity $^{18,21}$.

Fig. 3 represented the results of superoxide attenuation potential of Bauhinia variegata. Results were obtained in the order; stem $>$ flower $>$ leaves $>$ BHT $>$ ascorbic acid. The results showed that percentage inhibition of methanolic extracts of Bauhinia variegata was greater than standards. Stem exhibited maximum percentage inhibition of superoxide radical $(72.89 \pm 0.31 \%)$ while ascorbic acid $(49.11 \pm 0.24 \%)$ exhibited the least inhibition as compared to all other extracts. Thus stem of $B$. variegata contained maximum antioxidant potential. These results were according to their total phenolics contents. From current findings, it was concluded that percentage inhibition is concentration dependent. By increasing concentration, percentage inhibition increases. These results were in agreement with Shyur et al. ${ }^{22}$.

DPPH Radical attenuation potential: The DPPH free organic nitrogen radical is very stable, reacts with compounds that can donate hydrogen atoms and has a UV-visible absorption maximum at $515 \mathrm{~nm}$. The method is based on the scavenging of DPPH by antioxidants which upon a reduction reaction, decolourizes the deep purple DPPH methanol solution. The assay measures the reducing ability of antioxidants towards 


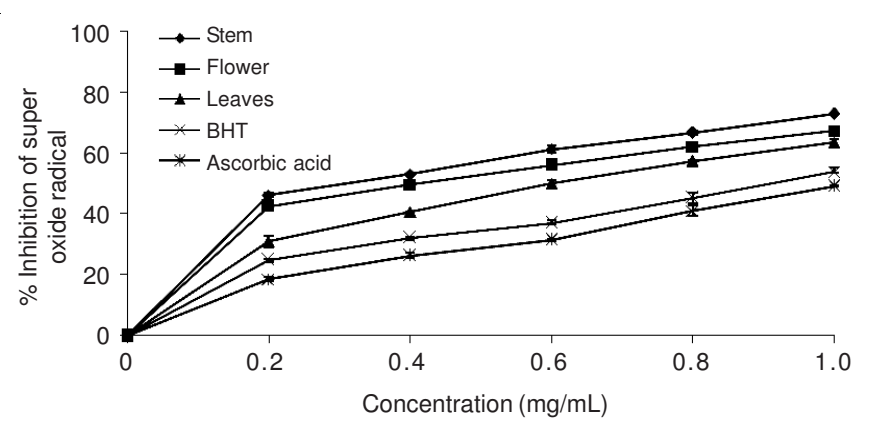

Fig. 3. Percentage inhibition of superoxide radical by different extracts of Bauhinia variegata

the DPPH radical using a UV-visible spectrophotometer. Alternatively, the antioxidant reducing ability can be evaluated by electron spin resonance. The greater the discolourations of the DPPH methanol solution then there will be lower the absorbance of the reaction mixture, thereby indicating significant free radical scavenging capacity ${ }^{23}$.

$$
\begin{gathered}
\mathrm{DPPH}^{\bullet}+\mathrm{HA} \\
\text { Purple }
\end{gathered} \underset{\text { Yellow }}{\mathrm{DPPHH}}+\mathrm{A}^{\bullet}
$$

In the present study the antioxidant activities of Bauhinia variegata was determined by DPPH scavenging assay. Many researchers also reported the use of DPPH scavenging assay for determination of antioxidant activity ${ }^{24,25}$. The DPPH scavenging activity of standards and different methanolic extracts of Bauhinia variegata has been shown in Fig. 4. The scavenging effect of plant extract and standard on the DPPH radical showed the following trend; ascorbic acid $>$ stem $>$ flower $>$ BHT > leaves. Results showed that the extracts of Bauhinia variegata showed lesser inhibition as compared to standard ascorbic acid but showed greater inhibition as compared to standard BHT. Results of current investigation showed decrease in absorbance with increase in concentration suggest that methanolic extracts of Bauhinia variegata has antioxidant activity due to its proton donating ability. These findings were in agreement with the previous studies ${ }^{24}$.

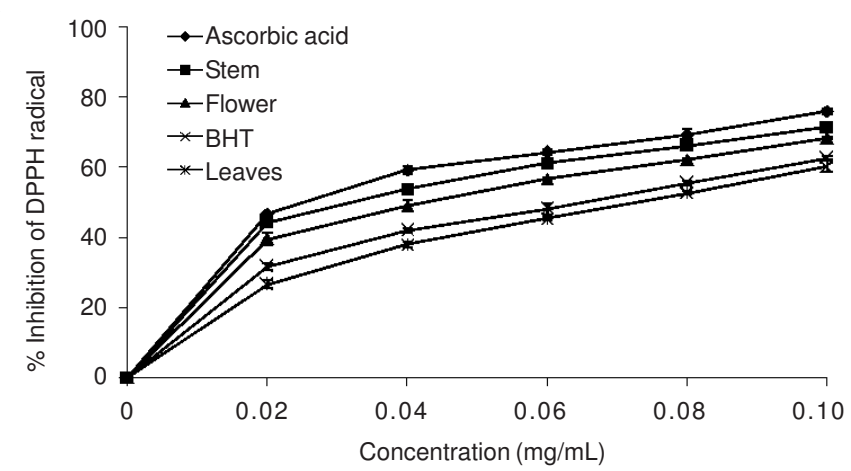

Fig. 4. Percentage inhibition of DPPH radical by different extracts of Bauhinia variegata

Percentage inhibition increases with increase in concentration that might be due to the increase in total phenolic contents. These results were in agreement with Masoko ${ }^{26}$.

Reducing potential assay: The reducing power of compounds could serve as a sign of probable antioxidant property. A noteworthy correlation was found between reduction potential and antioxidant activity ${ }^{10}$. Ferric ion reducing antioxidant power assay is employed to determine the reducing power due to its simplicity, speed and validity. In this assay, the electron-donating ability of an antioxidant is measured. Phenolic compounds show the reducing power due to their ability to donate electrons and also have ability to convert the ferric ion to ferrous ion ${ }^{27}$. Higher absorbance at high concentrations of investigated extracts indicates the strong reducing power potential.

In the present study the antioxidant potential of Bauhinia variegata was determined by reducing power assay. Fig. 5 represents the results of reducing power of various extracts. The reducing power activity ranged from $0.54 \pm 0.008(0.2$ $\mathrm{mg} / \mathrm{mL})$ to $1.976 \pm 0.045(1.0 \mathrm{mg} / \mathrm{mL})$. Among the parts of Bauhinia variegata, stem extract showed the highest reducing power $(1.976 \pm 0.045)$ followed by flower extract $(1.83 \% \pm$ $0.004)$ and leaves $(1.33 \pm 0.006)$. The extracts exhibited reducing power in the following manner; stem $>$ flower $>$ ascorbic acid $>$ BHT $>$ leaves. Extracts with highest total phenolic contents showed the highest reducing power. This proved the correlation between reducing power and total phenolic contents.

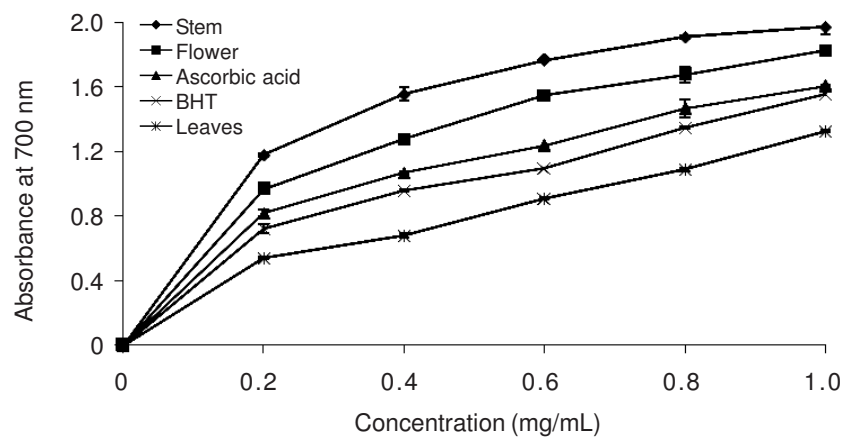

Fig. 5. Reducing potential of different parts of Bauhinia variegata

Antioxidant activity in linoleic acid system: When unsaturated fatty acids especially linoleic acid is present in the lipid membrane then this is the major cause of its oxidation. Inhibition of linoleic acid oxidation may indicate antioxidant activity. During the process of lipid peroxidation, there is formation of peroxides which oxidized $\mathrm{Fe}^{2+}$ to $\mathrm{Fe}^{3+}$. Then there is complex formation of $\mathrm{Fe}^{3+}$ with $\mathrm{SCN}^{-}$which shows maximum value of absorbance at $500 \mathrm{~nm}^{16}$.

In the present study, antioxidant activities at different time periods (24, 48 and $72 \mathrm{~h}$ ) were evaluated against linoleic acid peroxidation in the different parts of Bauhinia variegata. Many previous studies were reported about the use of this assay for the evaluation of antioxidant activity ${ }^{28-30}$. Results of the percentage inhibition of lipid peroxidation of different parts including stem, leaves and flowers of Bauhinia variegataare shown in Fig. 6. The inhibition capacity of linoleic acid oxidation of methanolic extracts of different parts of Bauhinia variegata was found to be lower than the inhibition capacity of the positive control (ascorbic acid and BHT). The order for percentage inhibition of lipid peroxidation was BHT $>$ ascorbic acid $>$ Stem $>$ Flower $>$ leaves.

After incubation of these extracts for 24,48 and $72 \mathrm{~h}$, the percentage inhibition of lipid peroxidation of Bauhinia variegata extracts of stem $(43.5 \pm 1.05 \%)$, flower $(38.17 \pm$ 
$0.98 \%)$ and leaves $(31 \pm 1.35 \%)$ respectively. As the time of incubation was increased, there was increase in the percentage inhibition of lipid peroxidation of the extracts. The results revealed that extract of stem contained significant antioxidant activity. Flower showed the moderate percentage inhibition. But leave showed the least percentage inhibition. These findings were according to their total phenolics contents. These findings were in agreement with previous researches ${ }^{31,32}$.

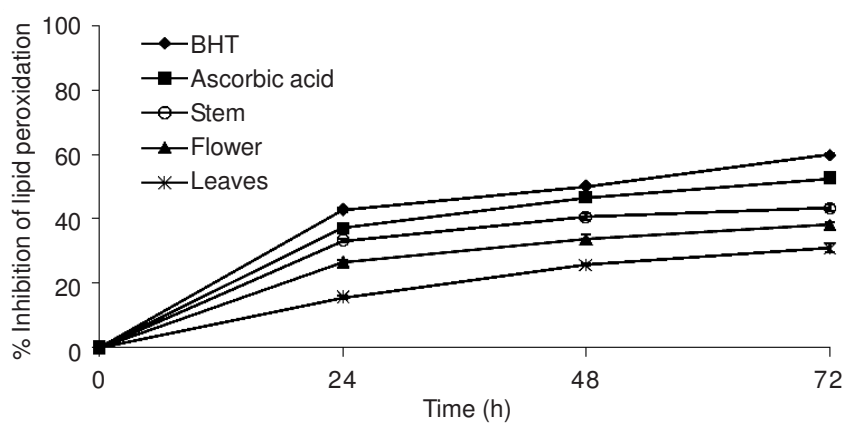

Fig. 6. Percentage inhibition of lipid peroxidation by different parts of Bauhinia variegata

\section{Conclusion}

Microwave assisted extraction is quickest technique for the extraction of polyphenols from plant materials. Stem of Bauhinia variegata possess highest amount of phenolic contents and antioxidant potential followed by flower and leaves.

\section{REFERENCES}

1. F.M. Awah and A.W. Verla, J. Med. Plants. Res., 4, 2482 (2010).

2. K.R. Nagulendran, S. Velavan, R. Mahesh and V.H. Begum, E-J. Chem., 4, 440 (2007).

3. R.R. Parmar, H.A. Bhuva, A.A. Joshi and R.B. Jadhav, Asian J. Chem., 22, 3851 (2010).

4. N. Gopinathan, K.K. Srinivasan and J.E. Mathew, Indian Drug, 41, 633 (2004).

5. S. Mcdonald, P.D. Prenzler, M. Autolovich and K. Robards, Food Chem., 73, 73 (2001).
6. R. Govindarajan, S. Rastogi and M. Vijayakumar, Biol. Pharma Bull., 26, 1424 (2003).

7. K.S. Vaidya, G.L.Viswanatha, C. Ramesh, K. Nandakumar and R. Srinath, J. Genet. Toxicol., 1, 1 (2008).

8. G.C. Yen and H.Y. Chen, J. Agric. Food Chem., 43, 27 (1995).

9. X. Duan, Y. Jiang, X. Su, Z. Zhang and J. Shi, Food Chem., 101, 1365 (2007).

10. T. Aydemir and S. Becerik, J. Food Biochem., 35, 65 (2011).

11. M. Gallo, R. Ferracane, G. Graziani, A. Ritieni and V. Fogliano, Mol., 15, 6365 (2010).

12. P.W. Tan, C.P. Tan and C.W. Ho, Int. Food Res. J., 18, 553 (2011).

13. B. Sultana, F. Anwar and M. Asharaf, Molecules, 14, 2167 (2009).

14. D. Komes, A.B. Cvitanovic, D. Horzic, G. Rusak, S. Likic and M. Berendika, Phytochem. Anal., 22, 172 (2011).

15. H.Y. Luo, W. Bin, Y.C. Guang and X.Y. Feng, Chinese Herbal Med., 2, 321 (2010).

16. E. Souri, G. Amin, H. Farsam, H. Jalalizadeh and S. Barezi, Iran. J. Pharma. Res., 7, 149 (2008).

17. Y. Sumanont, Y. Murakami, M. Tohda, O. Vajragupta, K. Matsumoto and H. Watanabe, Biol. Pharm. Bull., 27, 170 (2004).

18. G.P. Rajani and P. Ashok, Indian J. Pharmacol., 41, 227 (2009).

19. S. Shrikumar, M. Sukumar and T.K. Ravi, J. Nat. Rem., 7, 252 (2007).

20. S.S. Mishra, K.K. Patel, N. Raghuwanshi, A. Pathak, P.P. Panda, K. Girhepunje and C.N. Patro, Sch. Res. Lib., 2, 162 (2011).

21. A.D. Sathisha, H.B. Lingaraju and K. Sham Prasad, E-J. Chem., 8, 882 (2011).

22. L. Shyur, J. Tsung, J. Chen, C. Chiu and C. Lo, Int. J. Appl. Sci. Eng., 3, 195 (2005)

23. D. Huang, B. Ou and R.L. Prior, J. Agric. Food Chem., 53, 1841 (2005).

24. A.B. Aliyu, M.A. Ibrahim, A.M. Musa, H. Ibrahim, I.E. Abdulkadir and A.O. Oyewale, J. Med. Plants. Res., 3, 565 (2009).

25. V.K. Alluri, C.V. Rao, T.V.N. Rao, K.N. Reddy and G. Trimurtulu, Amer. J. Infect. Dis., 5, 64 (2009).

26. P. Masoko, S.S. Gololo, M.P. Mokgotho, J.N. Eloff, R.L. Howard and L.J. Mampuru, Afr. J. Tradit. Complement. Altern. Med., 7, 138 (2010).

27. M.Y. Shon, S.D. Choi, G.G. Kahng, S.H. Nam and N.J. Sung, Food Chem. Toxicol., 42, 659 (2004).

28. R. Akter, S.M.R. Hasan, S.A. Siddiqua, M.M. Majumder, M.M. Hossain, M.A. Alam, S. Haque and A. Ghani, J. Pharm. Sci., 1, 3 (2008).

29. M. Rai, G. Biswas, S. Chatterjee, S.C. Mandal and K. Acharya, E. J. Biol. Sci., 1, 39 (2009).

30. B. Sangameswaran, B.R. Balakrishnan, C. Deshraj and B. Jayakar, Pak. J. Pharm. Sci., 22, 368 (2009).

31. M. Elmastas, I. Gulcin, O. Isildak, O.I. Kufrevioglu, K. Ibaogluand and H.Y.A. Enein, J. Iran. Chem. Soc., 3, 258 (2006).

32. I. Gulcin, E. Kirecci, E. Akkemik, F. Topal and O. Hisar, Turk. J. Biol., 34, 175 (2010). 